\title{
SOME REMARKS ON RANKIN-COHEN BRACKETS OF EIGENFORMS
}

\author{
JABAN MEHER
}

\begin{abstract}
We investigate the cases for which products of two quasimodular or nearly holomorphic eigenforms are eigenforms. We also generalize the results of Ghate [5] to the case of Rankin-Cohen brackets.
\end{abstract}

\section{INTRODUCTION}

The space of modular forms of fixed weight on the full modular group has a basis of simultaneous eigenvectors for all Hecke operators. A modular form is called an eigenform if it is a simultaneous eigenvector for all Hecke operators. A natural question to ask is whether the product of two eigenforms (which may be of different weights) is an eigenform. The question was taken up by Duke [3] and Ghate [4. They proved that there are only finitely many cases where this phenomenon happens. Then a more general question i.e., the Rankin-Cohen bracket of two eigenforms was studied by Lanphier and Takloo-Bighash [8]. They also proved that except for finitely many cases, the Rankin-Cohen brackets of two eigenforms is not an eigenform. Recently, Beyerl, James, Trentacoste, Xue [1] have proved that this phenomenon extends to a certain class of nearly holomorphic modular forms. More explicitly, they have proved that there is only one more case apart from the cases listed in [3] and [4] for which the product of two nearly holomorphic eigenforms of certain type is a nearly holomorphic eigenform.

In this paper, we consider a few more cases of such results. First, we consider the product of two quasimodular eigenforms. Secondly, we consider the product of nearly holomorphic eigenforms. Finally, we generalize the result of Ghate [5] to the case of Rankin-Cohen brackets.

\section{Quasimodular Forms}

Let $\Gamma=S L_{2}(\mathbb{Z})$ be the full modular group and $\mathcal{H}$ denote the upper half plane. Let $M_{k}$ be the space of modular forms of weight $k$ on $\Gamma$.

Date: November 1, 2018.

2010 Mathematics Subject Classification. Primary 11F11, 11F25; Secondary 11F37.

Key words and phrases. Eigenforms, quasimodular forms, Maass-Shimura operator, RankinCohen brackets. 
Definition 2.1. A nearly holomorphic modular form $F$ of weight $k$ and depth $\leq$ $p$ on $\Gamma$ is a polynomial in $1 / y$ of degree $\leq p$ whose coefficients are holomorphic functions on $\mathcal{H}$ with moderate growth, such that $(c z+d)^{-k} F\left(\frac{a z+b}{c z+d}\right)=\left.F\right|_{k} \gamma=F$, where $\gamma=\left(\begin{array}{ll}a & b \\ c & d\end{array}\right) \in S L_{2}(\mathbb{Z})$.

Let $\widehat{M}_{k}^{(\leq p)}$ denote the space of such forms. We denote by $\widehat{M}_{k}=\cup_{p} \widehat{M}_{k}^{(\leq p)}$ the space of nearly holomorphic modular form of weight $k$ and $\widehat{M}_{*}=\oplus_{k} \widehat{M}_{k}$ the graded ring of all nearly holomorphic modular forms on $\Gamma$.

Definition 2.2. A quasimodular form of weight $k$ and depth $\leq p$ on $\Gamma$ is the constant term of a nearly holomorphic modular form of weight $k$ and depth $\leq p$ on $\Gamma$.

Let $\widetilde{M}_{k}^{(\leq p)}$ denote the space of such forms. Let $\widetilde{M}_{k}=\cup_{p} \widetilde{M}_{k}^{(\leq p)}$ be the space of quasimodular forms of weight $k$ and $\widetilde{M}_{*}=\oplus_{k} \widetilde{M}_{k}$ the graded ring of all quasimodular forms on $\Gamma$. Then it is known that $\widetilde{M}_{*}=\mathbb{C}\left[E_{2}, E_{4}, E_{6}\right]$. Here $E_{k}(z)=$ $1-\frac{2 k}{B_{k}} \sum_{m \geq 1} \sigma_{k-1}(m) q^{m}$ is the Eisenstein series of weight $k$, where $B_{k}$ is the $k$-th Bernoulli number, $\sigma_{k-1}(m)$ is the sum of $(k-1)$-th powers of the positive divisors of $m$, and $q=e^{2 \pi i z}$ with $z \in \mathcal{H}$. For more details on quasimodular forms see [2].

For $f \in \widetilde{M}_{k}$, define the action of $n^{t h}$ Hecke operator $T_{n}$ on $f$ by

$$
\left(T_{n} f\right)(z)=n^{k-1} \sum_{d \mid n} d^{-k} \sum_{b=0}^{d-1} f\left(\frac{n z+b d}{d^{2}}\right) .
$$

Then $T_{n}$ maps $\widetilde{M}_{k}$ to $\widetilde{M}_{k}$. A quasimodular form is said to be an eigenform if it is an eigenvector for all of the Hecke operators $T_{n}$ for $n \in \mathbb{N}$.

It is known that the differential operator $D=\frac{1}{2 \pi i} \frac{d}{d z}$ takes $\widetilde{M}_{k}$ to $\widetilde{M}_{k+2}$. We have the following proposition which follows by a similar argument as done in Proposition 2.4 and 2.5 of [1].

Proposition 2.3. If $f \in \widetilde{M}_{k}$, then $\left(D^{m}\left(T_{n} f\right)\right)(z)=\frac{1}{n^{m}}\left(T_{n}\left(D^{m} f\right)\right)(z)$, for $m \geq 0$. Moreover, we have $D^{m} f$ is an eigenform for $T_{n}$ iff $f$ is. In this case, if $\lambda_{n}$ is the eigenvalue of $T_{n}$ associated to $f$, then $n^{m} \lambda_{n}$ is the eigenvalue of $T_{n}$ associated to $D^{m} f$.

By comparing the constant coefficients of both sides of the equality given in Proposition 2.3 of [1], we get similar identies for the operator $D$. We now state two results which follow the same way as was done in [1]. 
Proposition 2.4. Suppose that $\left\{f_{i}\right\}_{i}$ is a collection of modular forms of distinct weights $k_{i}$. Then for $a_{i} \in \mathbb{C}^{*}, \sum_{i=1}^{t} a_{i} D^{\left(n-\frac{k_{i}}{2}\right)}\left(f_{i}\right)$ is an eigenform if and only if each $D^{\left(n-\frac{k_{i}}{2}\right)}\left(f_{i}\right)$ is an eigenform where the eigenvalues are the same for any $i$.

Proposition 2.5. If $k>l$ and $f \in M_{k}, g \in M_{l}$ are eigenforms, then for $r \geq 0$, $D^{\left(\frac{k-l}{2}+r\right)}(g)$ and $D^{r}(f)$ do not have the same eigenvalues.

Notation : For $k \in\{12,16,18,20,22,26\}$, let $\Delta_{k}$ denote the unique normalized cusp form of weight $k$ on $\Gamma$.

Using the above propositions and following the method as in [1], we have a result analogous to Theorem 3.1 of [1].

Theorem 2.6. Let $f \in M_{k}$ and $g \in M_{l}$ so that for some $r, s \geq 0$, $D^{r} f \in \widetilde{M}_{k+2 r}$ and $D^{s} g \in \widetilde{M}_{l+2 s}$ are eigenforms. Then $\left(D^{r} f\right)\left(D^{s} g\right)$ is an eigenform only in the following cases.

(1) The modular cases given in [3] and [4, namely

$$
\begin{aligned}
E_{4}^{2}= & E_{8}, E_{4} E_{6}=E_{10}, E_{6} E_{8}=E_{4} E_{10}=E_{14}, \\
E_{4} \Delta_{12}= & \Delta_{16}, E_{6} \Delta_{12}=\Delta_{18}, E_{4} \Delta_{16}=E_{8} \Delta_{12}=\Delta_{20}, \\
& E_{4} \Delta_{18}=E_{6} \Delta_{16}=E_{10} \Delta_{12}=\Delta_{22}, \\
E_{4} \Delta_{22}= & E_{6} \Delta_{20}=E_{8} \Delta_{18}=E_{10} \Delta_{16}=E_{14} \Delta_{12}=\Delta_{26} .
\end{aligned}
$$

(2) $\left(D E_{4}\right) E_{4}=\frac{1}{2} D E_{8}$.

Theorem 2.7. Let $f \in \widetilde{M}_{k}^{\leq p}$ and $g \in \widetilde{M}_{l}^{\leq q}$ be eigenforms such that $p, q<k / 2$. Then $f g$ is an eigenform only in the following cases.

(1) The modular cases given in [3] and [4], namely

$$
\begin{aligned}
E_{4}^{2}= & E_{8}, E_{4} E_{6}=E_{10}, E_{6} E_{8}=E_{4} E_{10}=E_{14}, \\
E_{4} \Delta_{12}= & \Delta_{16}, E_{6} \Delta_{12}=\Delta_{18}, E_{4} \Delta_{16}=E_{8} \Delta_{12}=\Delta_{20}, \\
& E_{4} \Delta_{18}=E_{6} \Delta_{16}=E_{10} \Delta_{12}=\Delta_{22}, \\
E_{4} \Delta_{22}= & E_{6} \Delta_{20}=E_{8} \Delta_{18}=E_{10} \Delta_{16}=E_{14} \Delta_{12}=\Delta_{26} .
\end{aligned}
$$

(2) $\left(D E_{4}\right) E_{4}=\frac{1}{2} D E_{8}$.

Proof. We know from Proposition 20 of [2] (page 59) that if $p<k / 2$, then $\widetilde{M}_{k}^{\leq p}=$ $\oplus_{r=0}^{p} D^{r}\left(M_{k-2 r}\right)$. Now, if $f \in \widetilde{M}_{k}^{\leq p}$ and $g \in \widetilde{M}_{l}^{\leq q}$ are eigenforms, then by Proposition 2.4 and 2.5, we can conclude that $f=D^{r}\left(f_{r}\right)$ and $g=D^{s}\left(g_{s}\right)$, for some $r, s$ and $f_{r} \in M_{k-2 r}, g_{s} \in M_{k-2 s}$. By applying the previous theorem, the result follows.

Remark 2.8. It is known from [2] that if $f$ is a non-zero quasimodular form of weight $k$ and depth $p$, then $p \leq k / 2$. 
Remark 2.9. If $f=\sum_{n \geq 1} a_{n} q^{n} \in \widetilde{M}_{k}$ is a non-zero eigenform, then $a_{1} \neq 0$. Thus, it follows that the product of two quasimodular eigenforms (having zero constant term) is not an eigenform.

It is easy to see that $E_{2}$ is an eigenform.

Remark 2.10. Following the same proof as in the case of $M_{k}$, one can prove that a quasimodular form in $\widetilde{M}_{k}$ with non-zero constant Fourier coefficient is an eigenform iff $f \in \mathbb{C} E_{k}$.

We have the following theorem.

Theorem 2.11. Let $f \in \widetilde{M}_{k}$ and $g \in \widetilde{M}_{l}$ be eigenforms such that the constant coefficients of both $f$ and $g$ are non-zero. Then $\left(D^{r} f\right)\left(D^{s} g\right)$ is an eigenform only in the following cases.

$$
E_{4}^{2}=E_{8}, E_{4} E_{6}=E_{10}, E_{6} E_{8}=E_{4} E_{10}=E_{14},\left(D E_{4}\right) E_{4}=\frac{1}{2} D E_{8} .
$$

To prove the above theorem, we first prove the following proposition.

Proposition 2.12. Let $f \in M_{k}$ be an eigenform. Then $E_{2} f$ is an eigenform if and only if $f \in \mathbb{C} \Delta_{12}$.

Proof. Since $D \Delta_{12}=E_{2} \Delta_{12}$, by Proposition 2.3 , $E_{2} \Delta_{12}$ is an eigenform.

Conversely, suppose that $E_{2} f$ is an eigenform with eigenvalues $\beta_{n}$, where $f=$ $\sum_{m \geq 0} a_{m} q^{m} \in M_{k}$ is an eigenform with eigenvalues $\lambda_{n}$. We know that $g=D f-$ $\frac{k}{12} E_{2} f \in M_{k+2}$. Then $T_{n}(D f)-\frac{k}{12} T_{n}\left(E_{2} f\right)=n \lambda_{n} D f-\frac{k}{12} n \lambda_{n} E_{2} f+\frac{k}{12}\left(n \lambda_{n}-\right.$ $\left.\beta_{n}\right) E_{2} f \in M_{k+2}$. Since $E_{2} f$ is not a modular form and $n \lambda_{n} D f-\frac{k}{12} n \lambda_{n} E_{2} f$ is a modular form, we have $n \lambda_{n}=\beta_{n}$ for all $n \geq 1$. Thus $g=D f-\frac{k}{12} E_{2} f \in M_{k+2}$ is an eigenform with eigenvalues $n \lambda_{n}$.

If $f=E_{k}$, then $g=\alpha E_{k+2}$ for some $\alpha \in \mathbb{C}$. Therefore, by applying $T_{n}$ to $\alpha E_{k+2}=D E_{k}-\frac{k}{12} E_{2} E_{k}$, we get for all $n \geq 1, n \sigma_{k-1}(n)=\sigma_{k+1}(n)$, which is not true.

If $f$ is a cusp form, without loss of generality assume that $f$ is normalized. Let $g=\sum_{m \geq 1} b_{m} q^{m}$. Since $b_{1}=1-\frac{k}{12}$, we have

$$
b_{n}=n a_{n}\left(1-\frac{k}{12}\right),
$$

for all $n \geq 1$. Now computing the values of $b_{n}$ from $D f-\frac{k}{12} E_{2} f$ in terms of $a_{n}$ and then substituting in the previous equation, we see that $a_{2}=-24, a_{3}=252$ and $a_{4}=-1472$. These are nothing but the second, third and fourth Fourier coefficients of $\Delta_{12}$ respectively. But Theorem 1 of [6] says that if $f_{1}$ and $f_{2}$ are two cuspidal 
eigenforms on $\Gamma_{0}(N)$ of different weights, then there exists $n \leq 4(\log (N)+1)^{2}$ such that $a_{n}\left(f_{1}\right) \neq a_{n}\left(f_{2}\right)$. Applying this theorem to $f_{1}=f, f_{2}=\Delta_{12}$ and $N=1$, we conclude that $k=12$. Thus we have $f=\Delta_{12}$.

Remark 2.13. Since $D E_{2}=\frac{E_{2}^{2}-E_{4}}{12}$ and $D E_{2}, E_{4}$ are eigenforms with different eigenvalues, $E_{2}^{2}$ is not an eigenform.

Proof of Theorem 2.11. By Theorem 2.6, Remark 2.9, Remark 2.10, Proposition2.12 and Remark 2.13, we only have to prove that in the following cases $\left(D^{r} E_{2}\right)\left(D^{s} E_{k}\right)$ is not an eigenform.

(1) $r=0$ and $s \geq 1$

(2) $r \geq 1$ and $s=0$.

For (1), let us assume on the contrary that $E_{2}\left(D^{s} E_{k}\right)$ is an eigenform where $s \geq$ 1. The first few coefficients of the normalized form $\frac{-B_{k}}{2 k} E_{2}\left(D^{s} E_{k}\right)=\sum_{n \geq 1} a_{n} q^{n}$ are $a_{1}=1, a_{2}=2^{s} \sigma_{k-1}(2)-24, a_{3}=3^{s} \sigma_{k-1}(3)-24\left(2^{s} \sigma_{k-1}(2)+3\right), a_{4}=4^{s} \sigma_{k-1}(4)-$ $24\left(3^{s} \sigma_{k-1}(3)+3 \cdot 2^{s} \sigma_{k-1}(2)+4\right)$.

Since $\frac{-B_{k}}{2 k} E_{2}\left(D^{s} E_{k}\right)$ is an eigenform we have $a_{4}=a_{2}^{2}-2^{k+2 s+1}$ and $a_{6}=a_{2} a_{3}$. Thus we have

$4^{s} \sigma_{k-1}(4)-24\left(3^{s} \sigma_{k-1}(3)+3 \cdot 2^{s} \sigma_{k-1}(2)+4\right)=2^{2 s} \sigma_{k-1}(2)^{2}-48 \cdot 2^{s} \sigma_{k-1}(2)+576-2^{k+2 s+1}$

and

$6^{s} \sigma_{k-1}(6)-24\left(5^{s} \sigma_{k-1}(5)+3 \cdot 4^{s} \sigma_{k-1}(4)+4 \cdot 3^{s} \sigma_{k-1}(3)+7 \cdot 2^{s} \sigma_{k-1}(2)+6\right)=\left(2^{s} \sigma_{k-1}(2)-\right.$ $24)\left(3^{s} \sigma_{k-1}(3)-24\left(2^{s} \sigma_{k-1}(2)+3\right)\right)$.

From the multiplicativity of $\sigma_{k-1}$ and $\sigma_{k-1}(4)=\sigma_{k-1}(2)^{2}-2^{k-1}$, these simplify to

$$
3^{s}\left(1+3^{k-1}\right)+2^{s}+28=2^{k+s-4}\left(2^{s}-2^{3}\right)
$$

and

(4) $5^{s} \sigma_{k-1}(5)+3^{s+1} \sigma_{k-1}(3)+2^{2 s+1} \sigma_{k-1}(2)^{2}+7 \cdot 2^{s+2} \sigma_{k-1}(2)-3 \cdot 2^{k+2 s-1}+78=0$.

Now, if $s \leq 3$, then the left hand side of (3) is positive, but the right hand side of the equation is non-positive. Thus $s \geq 4$. If $k \equiv 2(\bmod 4)$ and $s$ is odd, then $7+3^{s}\left(\frac{1+3^{k-1}}{4}\right) \equiv 2(\bmod 4)$, but $2^{k+s-6}\left(2^{s}-2^{3}\right)-2^{s-2}$ is divisible by 4 , giving a contradiction to (3). If $k \equiv 2(\bmod 4)$ and $s \equiv 0(\bmod 4)$, then $3^{s}\left(1+3^{k-1}\right)+2^{s}\left(1+2^{k-1}\right)+28 \equiv 0(\bmod 5)$, but 5 does not divide $2^{k+2 s-4}$. This gives a contradiction. If $k \equiv 2(\bmod 4)$ and $s \equiv 2(\bmod 4)$, then $3^{s+1} \sigma_{k-1}(3)+$ $2^{2 s+1} \sigma_{k-1}(2)^{2}+7 \cdot 2^{s+2} \sigma_{k-1}(2)-3 \cdot 2^{k+2 s-1}+78 \equiv 4(\bmod 5)$, but the remaining term of left hand side of (4) is divisible by 5 , giving a contradiction. If $k \equiv 0$ $(\bmod 4)$ and $s$ is even or $s \equiv 1(\bmod 4)$, then we get a contradiction from (3) and if $k \equiv 0(\bmod 4)$ and $s \equiv 3(\bmod 4)$, we get a contradiction from (44). This proves the theorem for case (1). 
For case (2), let us assume on the contrary that $\left(D^{r} E_{2}\right) E_{k}$ is an eigenform for $r \geq 1$. Let $\frac{-1}{24}\left(D^{r} E_{2}\right) E_{k}=\sum_{n \geq 1} b_{n} q^{n}$ be the normalized eigenform. The first few coefficients of the expansion are $b_{1}=1, b_{2}=3 \cdot 2^{r}-\frac{2 k}{B_{k}}, b_{3}=4 \cdot 3^{r}-\frac{2 k}{B_{k}}\left(3 \cdot 2^{r}+\sigma_{k-1}(2)\right)$, $b_{4}=7 \cdot 4^{r}-\frac{2 k}{B_{k}}\left(4 \cdot 3^{r}+3 \cdot 2^{r} \sigma_{k-1}(2)+\sigma_{k-1}(3)\right)$.

Since $\frac{-1}{24}\left(D^{r} E_{2}\right) E_{k}$ is a normalized eigenform, we have $b_{4}=b_{2}^{2}-2^{k+2 r+1}$. Substituting above values of $b_{2}$ and $b_{4}$ we get

$$
7 \cdot 4^{r}-\frac{2 k}{B_{k}}\left(4 \cdot 3^{r}+3 \cdot 2^{r} \sigma_{k-1}(2)+\sigma_{k-1}(3)\right)=\left(3 \cdot 2^{r}-\frac{2 k}{B_{k}}\right)^{2}-2^{k+2 r+1} .
$$

This can be simplified to

$$
\begin{gathered}
\left(\frac{2 k}{B_{k}}\right)^{2}+\frac{2 k}{B_{k}}\left(4 \cdot 3^{r}+3 \cdot 2^{r}\left(2^{k-1}-1\right)+1+3^{k-1}\right)+2^{2 r+1}\left(1-2^{k}\right)=0 . \\
\Rightarrow \frac{2 k}{B_{k}}=\frac{-b \pm \sqrt{b^{2}+2^{2 r+3}\left(2^{k}-1\right)}}{2}
\end{gathered}
$$

where

$$
b=4 \cdot 3^{r}+3 \cdot 2^{r}\left(2^{k-1}-1\right)+1+3^{k-1} .
$$

Since $\frac{2 k}{B_{k}}$ is a rational number, $b^{2}+2^{2 r+3}\left(2^{k}-1\right)$ is a perfect square, and since 2 divides $b, \frac{2 k}{B_{k}}$ is an integer. This implies that $k \in\{2,4,6,8,10,14\}$. Since the case $k=2$ is shown in case (1), we only consider $k \in\{4,6,8,10,14\}$.

Let $k=4$. In this case, $\frac{2 k}{B_{k}}=-240$. Since $\frac{2 k}{B_{k}}$ is negative, from (15), we get

$$
\begin{aligned}
& -b-\sqrt{b^{2}+2^{2 r+3}\left(2^{4}-1\right)}=-480 \\
\Rightarrow & b^{2}+15 \cdot 2^{2 r+3}=(b-480)^{2} \\
\Rightarrow & b=240-2^{2 r-3}
\end{aligned}
$$

Substituting this value of $b$ in (마), we get

$$
2^{2 r-3}+4 \cdot 3^{r}+21 \cdot 2^{r}-212=0 .
$$

Now, we can see that (17) is not satisfied for any positive integer $r$, giving a contradiction. The other cases are done similarly, whereby one uses (5) to obtain an equation in terms of $r$. It is straightforward to show that this equation cannot be satisfied for any appropriate integer values of $r$. This concludes the proof of the theorem.

Corollary 2.14. Let $f \in M_{k}$ be an eigenform. Then $\left(D^{r} E_{2}\right) f$ is an eigenform if and only if $r=0$ and $f \in \mathbb{C} \Delta_{12}$.

Proof. It is a direct consequence of Remark 2.9, Theorem 2.11 and Proposition 2.12. 


\section{NEARLY hOLOMORPHIC MODULAR FORMS}

Definition 3.1. The Maass-Shimura operator $\delta_{k}$ on $f \in \widehat{M}_{k}$ is defined by

$$
\delta_{k}(f)=\left(\frac{1}{2 \pi i}\left(\frac{k}{2 i \operatorname{Im}(z)}+\frac{\partial}{\partial z}\right) f\right)(z) .
$$

The operator $\delta_{k}$ takes $\widehat{M}_{k}$ to $\widehat{M}_{k+2}$. Here we consider the action of $\delta_{k}$ on $M_{k}$. The operator $T_{n}$, for each $n \geq 1$ as defined by (11), maps $\widehat{M}_{k}$ to $\widehat{M}_{k}$. The function $E_{2}^{*}(z)=E_{2}(z)-\frac{3}{\pi \operatorname{Im}(z)}$ is a nearly holomorphic modular form of weight 2 on $\Gamma$ and it is also an eigenform.

Theorem 3.2. Let $f$ be a normalized eigenform in $M_{k}$. Then $E_{2}^{*} f$ is an eigenform if and only if $f=\Delta_{12}$.

Proof. It is known from Proposition 2.5 of [1] that $\delta_{12}\left(\Delta_{12}\right)=E_{2}^{*} \Delta_{12}$ is an eigenform. For any modular form $f \in M_{k}$, we have $\delta_{k}(f)-\frac{k}{12} E_{2}^{*} f=D f-\frac{k}{12} E_{2} f \in M_{k+2}$. Now assume that $f \in M_{k}$ is a normalized eigenform such that $E_{2}^{*} f$ is an eigenform. Then proceeding as in Proposition 2.12, we conclude that $f=\Delta_{12}$.

\section{RANKin-COHEn Brackets of HOLOMORPhiC EIGENFORMS}

Let $M_{k}\left(\Gamma_{1}(N)\right), S_{k}\left(\Gamma_{1}(N)\right)$ and $\mathcal{E}_{k}\left(\Gamma_{1}(N)\right)$ be respectively the spaces of modular forms, cusps forms and Eisenstein series of weight $k \geq 1$ on $\Gamma_{1}(N)$, and let $M_{k}(N, \chi)$, $S_{k}(N, \chi), \mathcal{E}_{k}(N, \chi)$ be the spaces of modular forms, cusps forms and Eisenstein series of level $N$ and character $\chi$ respectively. We have an explicit basis $\mathcal{B}$ for $M_{k}\left(\Gamma_{1}(N)\right)$ which consist of common eigenforms for all Hecke operators $T_{n}$ with $(n, N)=1$ as described in [5]. An element of $M_{k}\left(\Gamma_{1}(N)\right)$ is called an almost everywhere eigenform or a.e. eigenform for short, if it is constant multiple of an element of $\mathcal{B}$. For further details see [5].

Let $g \in M_{k_{1}}(N, \chi)$ and $h \in M_{k_{2}}(N, \psi)$. The $m^{\text {th }}$ Rankin-Cohen bracket of $f$ and $g$ is defined by

$$
[g, h]_{m}(z)=\sum_{r+s=m}(-1)^{r}\left(\begin{array}{c}
m+k_{1}-1 \\
s
\end{array}\right)\left(\begin{array}{c}
m+k_{2}-1 \\
r
\end{array}\right) g^{(r)}(z) h^{(s)}(z),
$$

where $g^{(r)}(z)=D^{r} g(z)$ and $h^{(s)}(z)=D^{s} h(z)$.

It is known that $[g, h]_{m} \in M_{k_{1}+k_{2}+2 m}(N, \chi \psi)$ and $[g, h]_{m}$ is a cusp form if $m \geq 1$.

For $k>2$, the Eisenstein series is defined by

$$
E_{k}^{(N, \psi)}(z)=\sum_{\gamma \in \Gamma_{\infty} \backslash \Gamma_{0}(N)} \bar{\psi}(d)(c z+d)^{-k} \in \mathcal{E}_{k}(N, \psi)
$$


where $z \in \mathcal{H}$ and the sum varies over all $\gamma=\left(\begin{array}{ll}a & b \\ c & d\end{array}\right) \in \Gamma_{0}(N)$ modulo $\Gamma_{\infty}=\left\{\left(\begin{array}{ll}1 & n \\ 0 & 1\end{array}\right) \mid n \in \mathbb{Z}\right\}$. We recall Proposition 6 of [11]:

Theorem 4.1. Let $k_{1}, k_{2}, m$ be integers satisfying $k_{2} \geq k_{1}+2>2$ and let $k=$ $k_{1}+k_{2}+2 m$. If $f(z)=\sum_{n=1}^{\infty} a_{n} q^{n} \in S_{k}(N, \chi \psi)$ and $g(z)=\sum_{n=0}^{\infty} b_{n} q^{n} \in M_{k_{1}}(N, \chi)$, then $\left\langle f,\left[g, E_{k_{2}}^{(N, \psi)}\right]_{m}\right\rangle=\frac{\Gamma(k-1) \Gamma\left(k_{2}+m\right)}{(4 \pi)^{k-1} m ! \Gamma\left(k_{2}\right)} \sum_{n=1}^{\infty} \frac{a_{n} \bar{b}_{n}}{n^{k_{1}+k_{2}+m-1}}$, where $\langle$,$\rangle is the Petersson inner$ product.

Now, for an arbitrary positive integer $N$, let $Q \mid N$ such that $(Q, N / Q)=1$. Let $W_{Q}$ be the Atkin-Lehner operator on $M_{k}(N, \chi)$. Let $\chi=\chi_{Q} \chi_{N / Q}$. Then it is known that $W_{Q}$ maps $M_{k}\left(N, \chi_{Q} \chi_{N / Q}\right)$ to $M_{k}\left(N, \bar{\chi}_{Q} \chi_{N / Q}\right)$ and it is an involution. It takes cusp forms to cusp forms and a.e. eigenforms to a.e. eigenforms (see [9] for details). We have the following lemma (see [7], Proposition 1).

Lemma 4.2. If $g \in M_{k_{1}}(N, \chi)$ and $h \in M_{k_{2}}(N, \psi)$, then $[g, h]_{m} \mid W_{Q}=\left[g\left|W_{Q}, h\right| W_{Q}\right]_{m}$.

Let $\psi_{i}$ be Dirichlet characters $\bmod M_{i}, i=1,2$ such that $\psi_{1} \psi_{2}(-1)=(-1)^{k}$, where $k \geq 1$. Also assume that:

(1) if $k=2$ and $\psi_{1}$ and $\psi_{2}$ both are trivial, then $M_{1}=1$ and $M_{2}$ is a prime number,

(2) otherwise, $\psi_{1}$ and $\psi_{2}$ are primitive characters.

Put $M=M_{1} M_{2}$ and $\psi=\psi_{1} \psi_{2}$.

Let $f_{k}\left(Q z, \psi_{1}, \psi_{2}\right)$, where $Q M_{1} M_{2} \mid N$ be the set of elements of $\mathcal{E}_{k}(N, \psi)$ as given in Theorems 4.7.1 and 4.7.2 of [10] which form a basis of common eigenforms for all the Hecke operators $T_{n}$ of level $N$, with $(n, N)=1$.

Remark 4.3. Using Theorem 4.1 and following the lines of Proposition 3 of [5], we have the following:

For positive integers $k, k_{1}, k_{2}, m$ satisfying $k_{2} \geq k_{1}+2>2$ and $k=k_{1}+k_{2}+2 m$, $g \in S_{k_{1}}(N, \chi)$ an a.e. eigenform which is a newform, $h=E_{k_{2}}^{(N, \psi)} \in \mathcal{E}_{k_{2}}(N, \psi)$, if dim $S_{k}^{\text {new }}(N, \chi \psi) \geq 2$ then $[g, h]_{m}$ is not an a.e. eigenform.

Remark 4.4. Similarly as mentioned in the previous remark, we have an analogous result to Proposition 4 of [5] in this case:

For positive integers $k, k_{1}, k_{2}, m$ satisfying the same condition as in the previous remark, $g=f_{k_{1}}\left(z, \chi_{1} \chi_{2}\right)$ an a.e. eigenform as described above with $\chi$ primitive, $h=E_{k_{2}}^{(N, \chi)} \in \mathcal{E}_{k_{2}}(N, \chi)$, if $\operatorname{dim} S_{k}^{\text {new }}(N, \chi \psi) \geq 2$ then $[g, h]_{m}$ is not an a.e. eigenform.

Theorem 4.5. Let $k_{1}, k_{2}, k, m$ be positive integers such that $k=k_{1}+k_{2}+2 m$ and let $N$ be square-free. 
(i) If $g \in S_{k_{1}}\left(\Gamma_{1}(N)\right)$ and $h \in S_{k_{2}}\left(\Gamma_{1}(N)\right)$ are a.e. eigenforms, then $[g, h]_{m}$ is not an a.e. eigenform.

(ii) Let $k_{1} \geq 3$ and $k_{2} \geq k_{1}+2>2$. Suppose that $g \in S_{k_{1}}(N, \chi)$ is an a.e. eigenform which is a newform and $h \in \mathcal{E}_{k_{2}}(N, \psi)$. If $\operatorname{dim} S_{k}^{\text {new }}(N, \chi \psi) \geq 2$, then $[g, h]_{m}$ is not an a.e. eigenform.

(iii) Let $k_{1}, k_{2} \geq 3,\left|k_{1}-k_{2}\right| \geq 2$. Let $g=f_{k_{1}}\left(z, \chi_{1}, \chi_{2}\right) \in \mathcal{E}_{k_{1}}(N, \chi)$ and $h=$ $f_{k_{2}}\left(z, \psi_{1}, \psi_{2}\right) \in \mathcal{E}_{k_{2}}(N, \psi)$ be a.e. eigenforms as mentioned above with $\chi$ and $\psi$ primitive characters. If $\operatorname{dim} S_{k}^{\text {new }}(N, \chi \psi) \geq 2,[g, h]_{m}$ is not an a.e. eigenform.

Proof. Assume that

$$
[g, h]_{m}=f
$$

is an a.e. eigenform. Then $f(z)=f_{0}(Q z)$, where $M|N, Q|(N / M)$ and $f_{0} \in S_{k}(M, \chi)$ is a normalized newform. Since $N$ is square-free, for any divisior $Q$ of $N,(Q, N / Q)=$ 1. We also have $f \mid W_{Q}=Q^{-k / 2} \chi(w) f_{0}(z)$. Applying the operator $W_{Q}$ to (8) and by Lemma 4.2, we get $\left[g\left|W_{Q}, h\right| W_{Q}\right]_{m}=f \mid W_{Q}=$ const. $f_{0}$. This gives a contradiction since the $q$-expansion of $f_{0}$ (being primitive) starts with $q$, whereas the $q$-expansion of $\left[g\left|W_{Q}, h\right| W_{Q}\right]_{m}$ starts with at least $q^{2}$. This proves (i).

Let $h=f_{k_{2}}\left(Q z, \psi_{1}, \psi_{2}\right)$, for $Q \mid\left(N / M_{1} M_{2}\right)$. Since $N$ is square-free, for any divisor $Q$ of $N$, we have $(Q, N / Q)=1$. Now applying $W_{N / Q M_{2}}$ on $h$ and using Proposition 1 and Lemma 1 of [5], we get $h \mid W_{N / Q M_{2}}=$ const. $f_{k_{2}}\left(\frac{N z}{M_{1} M_{2}}, \psi_{0}, \bar{\psi}_{1} \psi_{2}\right)=$ const. $E_{k_{2}}^{\left(N, \bar{\psi}_{1} \psi_{2}\right)}$, where $\psi_{0}$ is the principal character. Now assume on the contrary that $[g, h]_{m}$ is an a.e. eigenform. Applying $W_{N / Q M_{2}}$ to $[g, h]_{m}$ and using Lemma 4.2, we see that $\left[g\left|W_{N / Q M_{2}}, h\right| W_{N / Q M_{2}}\right]_{m} \in S_{k}\left(N, \chi_{Q M_{2}} \bar{\chi}_{N / Q M_{2}} \bar{\psi}_{1} \psi_{2}\right)$ is an a.e. eigenform. Since the $W$-operator is an isomorphism and takes a newform space to a newform space, $\operatorname{dim} S_{k}\left(N, \chi_{Q M_{2}} \bar{\chi}_{N / Q M_{2}} \bar{\psi}_{1} \psi_{2}\right) \geq 2$. Then applying Remark 4.3 to $g \mid W_{N / Q M_{2}} \in S_{k}\left(N, \chi_{Q M_{2}} \bar{\chi}_{N / Q M_{2}}\right)$ and $h \mid W_{N / Q M_{2}}=$ const. $E_{k_{2}}^{\left(N, \bar{\psi}_{1} \psi_{2}\right)}$, we get a contradiction. This proves (ii).

If $k_{2}-k_{1} \geq 2$, then as in the proof of (ii), we apply the operator $W_{N / M_{2}}$ to $h$ and $g$ and we get $h \mid W_{N / M_{2}}=$ const. $E_{k_{2}}^{\left(N, \bar{\psi}_{1} \psi_{2}\right)}$ and $g \mid W_{N / M_{2}}$ is a form with primitive character. Applying Remark 4.4, we get (iii). If $k_{1}-k_{2} \geq 2$, then interchanging the roles of $g$ and $h$ gives the required result.

\section{Acknowledgments}

I thank D. Lanphier for giving useful informations about his papers. I thank B. Ramakrishnan for useful discussions and making numerous suggestions. I also thank Sanoli Gun for her useful comments. Finally, I thank the referee for meticulously reading the manuscript and making numerous suggestions which improved the presentation. 


\section{REFERENCES}

[1] J. Beyerl, K. James, C. Trentacoste, H. Xue, Products of nearly holomorphic eigenforms, Ramanujan J. 27 (2012), 377-386.

[2] J. H. Bruinier, G. van der Geer, G. Harder, D. Zagier, The 1-2-3 of Modular Forms, Springer, 2008.

[3] W. Duke, When is the product of two Hecke eigenforms an eigenform ?, In Number theory in progress, Vol. 2 (Zakopane-Kościelisko, 1997), de Gruyter, Berlin,(1999), 737-741.

[4] E. Ghate, On monomial relations between Eisenstein series, J. Ramanujan Math. Soc. 15, (2000), 71-79.

[5] E. Ghate, On products of eigenforms, Acta Arith. 102 (2002), 27-44.

[6] A. Ghitza, Distinguishing Hecke eigenforms, Int. J. Number Theory 7 (2011), No. 5, 12471253.

[7] D.Lanphier, Combinatorics of Maass-Shimura operators, J. Number Theory 128 (2008), no.8, 2467-2487.

[8] D. Lanphier, R. Takloo-Bighash, On Rankin-Cohen brackets of eigenforms, J. Ramanujan Math. Soc. 19 (2004), no. 4, 253-259.

[9] W. W. Li, Newforms and functional equations, Math. Ann. 212 (1975), 285-315.

[10] T. Miyake, Modular Forms, Springer, 1989.

[11] D.Zagier, Modular forms whose Fourier coefficients involve zeta-functions of quadratic fields, in Modular Functions of One Variable IV, Lect. Notes in Math. 627, Springer, Berlin, 1977, $105-169$.

Harish-Chandra Research Institute, Chhatnag Road, Jhunsi, Allahabad 211019 , INDIA.

E-mail address: jaban@hri.res.in 